\title{
Differenz
}

Revista internacional de estudios heideggerianos y sus derivas contemporáneas

AÑO 8, NÚMERO 7: JULIO DE 2021. ISSN 2695-9011 - e-ISSN 2386-4877 - DOI: 10.12795/Differenz.2021.i07.02 [pp. 25-48]

Recibido: $30 / 03 / 2021$

Aceptado: 10/06/2021

\section{La barbarie de Michel Henry: De una filosofía epitelial a la cuestión de la técnica.}

\section{Michel Henry's La barbarie: From an epithelial philosophy to the question of technology.}

\section{Gaston Giribet}

Universidad de Buenos Aires-CONICET

\section{Resumen:}

En el primer encuentro con La barbarie (Michel Henry, 1987), y sin que esta afirmación pretenda ser general o de manera alguna esconder los prejuicios individuales al acercarse a una lectura de esta naturaleza, surge en muchos de nosotros la pregunta de si se está frente a una pieza de irracionalismo ingenuo, una paráfrasis pesimista de cuestiones ya tratadas, por ejemplo, en Die Frage nach der Technik (Heidegger, 1954), o si, por el contrario, Henry ofrece en este libro una visión radicalmente renovadora del riesgo en que se encuentra la cultura (y lo que él define como vida) ante el avance de una ideología, la de la barbarie, que promulga el saber de la ciencia como el único saber. Responder a esta pregunta es, precisamente, la motivación principal de este trabajo. Suspendiendo los prejuicios sobre el libro de Henry, incluso a riesgo de perder en el camino mucho de la inocultable pretensión de provocación que es parte inextirpable de esta obra suya, nos 
avocamos a señalar los elementos esenciales discutidos en La barbarie, a encontrar las resonancias que éstos pueden tener con el resto de la obra de Henry, y, en especial, a discutir los temas principales de La barbarie en relación a otro texto clave de la tradición fenomenológica: las consideraciones de Heidegger en torno a La pregunta por la técnica.

Palabras clave: Fenomenología; Heidegger; Filosofía de la técnica; Michel Henry; Epistemología.

\section{Abstract:}

In the first encounter with La barbarie (Michel Henry, 1987), and without the statement pretending to be general or in any way to hide individual prejudices when approaching a text of this sort, the question arises in many of us as to whether one is before a piece of naive irrationalism, a pessimistic paraphrase of issues already treated, for example, in Die Frage nach der Technik (Heidegger, 1954), or if, on the contrary, Henry offers in this book a radically renewed vision of the risk that culture (and what he defines as life) is in before the advance of an ideology, that of barbarism, which promulgates the knowledge of science as the only knowledge. Answering this question is precisely the main motivation for this work. Suspending prejudices about Henry's book, even at the risk of losing along the way much of the undeniable pretense of provocation that is an inextricable part of this work of him, we set out to point out the essential elements discussed in La barbarie, to find the resonances that these may have with the rest of Henry's work, and, in particular, to discuss the main themes of La barbarie in relation to another key text of the phenomenological tradition: Heidegger's considerations on The question concerning technology.

Keywords: Phenomenology, Heidegger, Philosophy of technology; Michel Henry; Epistemology.

\section{Introducción}

\subsection{Hacia una investigación del aparecer puro}

Mientras que otros fenomenólogos contemporáneos, incluso aquellos conocidos por ahondar en lo que es basal de la fenomenología, como Jean-Luc Marion, profundizaron en la manifestación del fenómeno a partir de una intención original de carácter expositivo y ordenador de las ideas de Husserl, Henry se yergue ante la fenomenología husserliana -y dentro de ella- con una actitud crítica que nace de un reclamo inicial y legítimo: "Ha llegado ya el momento de que la filosofía deje de pensar el sujeto bajo las categorías de objeto y 
piense realmente la subjetividad misma"1. Concediéndole a Husserl su intuición genial y reconociéndole "el extraordinario vuelco operado por [su] fenomenología", en especial en los temas relacionados con los que trata en La barbarié2, Henry no duda en demarcar los límites de la fenomenología de Husserl ni duda en someter a embate crítico la estructura fundamental de la misma al momento de disputarle a la conciencia de mundo su lugar preponderante. Por eso decimos que la fenomenología de Henry tiene una pretensión fundacional: "Husserl no ha sido lo suficientemente radical". En la introducción a la edición en castellano de La barbarie, Moratalla dice sobre el trabajo de Henry: "no hay un 'giro' operado en la fenomenología (...) sino una radicalización"', y agrega: "[s]u filosofía nace en el interior de la fenomenología, pero discute su presuposición fundamentadora. (...) [S]e pregunta (...) la esencia de la manifestación. Es una investigación (...) del aparecer puro (...) sin la consideración de un objeto"4.

\subsection{Michel Henry}

Michel Henry nació en 1922 en la Indochina francesa, y vivió allí su primera infancia. En su juventud, ya en la Francia continental, estudió filosofía en París, en la École Normale Supérieure, dependiente por aquel entonces de la Université de Paris. En 1963 presentó su tesis doctoral, L'essence de la manifestation, que luego se convertiría en el primero de sus libros más celebrados.

La filosofía de Henry, y a menudo se insiste en ello como si fuese necesario hacerlo, es una fenomenología, una "fenomenología de la vida". Formando parte de lo que podríamos llamar una segunda recepción de la fenomenología en Francia, una ya no limitada a la tarea de generalización del alcance del método fenomenológico, sino avocada a una reconsideración radical de las bases mismas de la fenomenología, Henry opera un giro en la teoría de Husserl: Mientras que el fenómeno es entendido en la fenomenología husserliana como aquello que aparece en el mundo, en lo que Henry denomina "la exterioridad", para Henry, Husserl sigue prisionero ${ }^{5}$ del presupuesto clásico de que

1 Domingo Moratalla, T. "Introducción", en Henry M. La barbarie. Caparrós, Madrid, 2006, p. 13. 2 HENRY M. Op. cit., p. 22.

3 Domingo Moratalla, T. Op. cit., p. 8.

4 lb., p. 9.

5 Señala Bernet que "Husserl nunca llegó a decir, como lo ha hecho Michel Henry, que la esencia de la subjetividad es una afectividad sin ninguna conexión inherente con una $u$ otra forma de intencionalidad" (BERNET, R. "An intentionality without subject or object?", en Man and World 27, 1994, p. 232); "Henry (...) afirm[a] que la vida del sujeto nunca puede aparecer en la forma de la trascendencia de un objeto intencional" (Ib., p. 238), y afirma que "el carácter intensamente 
aquello que se nos presenta lo hace distinto de nosotros y es, entonces, pensado siempre en una "exterioridad"6.

Henry contesta a la concepción husserliana de fenómeno con la de una "fenomenología radical de la vida"; una vida que él define, asimismo, desde una perspectiva fenomenológica: aquello que posee la facultad y el poder de "sentir y experimentarse en cada punto de su ser", según diría en La barbarie. La vida es, según Henry, fuerza y afecto, un oscilar perpetuo entre sufrimiento y alegría que se experimenta a sí mismo y es invisible; no puede verse desde la exterioridad del mundo, sino sólo desde la interioridad. Estas nociones germinan ya en su obra de los años sesenta, pero reaparecen con fuerza en los trabajos posteriores; en particular en La barbarie, sobre la que nos ocuparemos aquí, pero también en su trabajo posterior: pensemos en Encarnación (2000) o incluso en su trabajo anterior sobre Kandinsky, Ver lo invisible (1988), donde las dimensiones de lo visible y lo invisible aparecen atravesando la unidad primitiva del ser:

El ser no es, pues, una noción unívoca. Dos dimensiones lo atraviesan y vienen a desgarrar su unidad primitiva (...): la de lo visible, donde la luz del mundo las cosas se dan a nosotros y son vividas por nosotros como fenómenos exteriores; la de lo invisible, donde, en ausencia de ese mundo y de su luz, antes incluso de que surgiese ese horizonte de exterioridad que pone todo a cierta distancia de nosotros y nos lo pro-pone a título de ob-jeto (...), la vida se ha apoderado ya de su ser propio, abrazándose a sí misma en esa prueba interior e inmediata de sí que es su pathos, que hace de ella la vida?.

Un año antes de Ver lo invisible publica La barbarie, en 1987. En un momento en el que la hipótesis de dos formas de mundo posibles sufría el crujir de sus grietas y la presencia premonitoria de la unicidad de discursos, Henry escribía sobre el abismo al que nos enfrentábamos debido a la ideología que nos presentaba una única forma de verdad, la verdad de la ciencia galileana y su abstracción del mundo; la ideología de la barbarie, que viene a someter a las otras formas de saber, la de la religión, la del arte, la de la cultura, mas ya no meramente subordinándolas sino poniéndolas en riesgo de desaparecer para jamás regresar.

subjetivo de los sentimientos excluye completamente la posibilidad de que todavía puedan ser considerados como experiencias vividas intencionalmente" (Ib., p. 241).

6 Domingo Moratalla, T. Op. cit., p. 8.

7 Henrr, M. Ver lo invisible. Siruela, Madrid, 2008, p. 19. 


\subsection{Una fenomenología epitelial}

Para Henry, la vida es la verdad. Podemos ver en esto la inversión realizada por Marx devenida dictum: "Es la vida de los hombres la que determina la consciencia". Según Henry, vivir significa ser. Se trata de una ontología de la vida donde la vida es lo primero, lo absolutamente cierto, lo evidente para todo viviente, para todo el que es. Pero no se trata de un sentio ergo sum en tanto la remisión a la conciencia es, justamente, lo que no media aquí.

Henry sostiene que la filosofía no había pensado la vida, y he aquí el rasgo de pretensión fundacional de su fenomenología. Este olvido de la vida se debe, según él, a un equívoco inicial en la filosofía occidental: la caracterización del ser en términos de la exterioridad. La verdad de la vida, para Henry, está en la "ipseidad", en el sentirse a sí mismo, en el pathos. Una vez más, la vida es la verdad.

La vida es, para Henry, predominantemente praxis. El sujeto arrojado fuera de la vida es mera representación. Esto es importante en especial para entender La barbarie, dado que la ciencia galileana que la ideología de la barbarie instaura como única forma de saber tiene el rasgo definitorio de abstracción del mundo, de mera representación de éste; rasgo que, por otro lado, está grabado a fuego en la ciencia moderna en tanto nace de su pretensión de universalidad, de generalidad y de objetividad. De la universalidad nace la mera representación como pobre reflejo de la vida; de la objetividad nace la violencia ejercida sobre lo subjetivo. Toda metafísica de la representación -e.g. la kantiana como ejemplo antonomástico- está en contra de una concepción con el centro en la ipseidad. El paradigma de la conciencia, la autoconciencia, la reflexión no están primeros; no son lo primero; lo primero es la autoafectividad: "la vida se siente y se experimenta en sí misma", dirá Henry. Este es su ab initio: La autoafección es primera e inmediata; la afectividad constituye la esencia de la afección. El ser, y sin que esto deba entenderse como un exhorto a una mera reinstauración de un canon entre lo apotético y lo paratético, debe ser pensado en términos de esa interioridad. En este sentido, podemos llamar a la de Henry una filosofía epitelial. Con la introducción del término epitelial queremos hacer referencia a la afirmación de la interioridad, al cuerpo en el sentido más íntimo; ese tejido interno del organismo que reviste las cavidades del cuerpo y sus órganos.

Henry propone, como otros antes que él, una vuelta al sujeto; pero la suya es una vuelta radical, reaccionaria, contrarreformista: Si los que él denomina "ismos" hubieron desterrado al sujeto de la filosofía de fines del siglo XIX y comienzos del XX, Henry emprende una crítica a la crítica del sujeto. La subjetividad es afirmada en Henry con una 
fuerza inusitada; con una fuerza que es propia de pilares basales y robustos, y es que él erige sobre esta idea todo su edificio filosófico.

\subsection{La barbarie}

Moratalla escribe sobre Henry:

Henry se va a detener en la dimensión de inmanencia radical donde el aparecer se presenta a sí mismo. (...) Esta inmanencia absoluta (...) es la autoafección (...), no conoce ninguna objetividad, ninguna alteridad. Esta posibilidad de autoafección es (...) la esencia de la ipseidad, de los que somos, y que Henry llamaba "vida"8.

\section{Y agrega:}

Al igual que la subjetividad no es sólo conciencia, intencionalidad, relación a un objeto, (...) el cuerpo no es sólo acción, sino más fundamentalmente pathos, pasión; reconocimiento de una pasividad radical. La filosofía de un cuerpo subjetivo radicalmente inmanente será la base para una crítica del dualismo cartesiano (...) De ahí el sentido de convertir una fenomenología radical en una fenomenología material ${ }^{9}$.

La barbarie denuncia, como decíamos, la unicidad del saber científico, de la concepción tecnocrática del mundo; la ideología de la barbarie es la instauración del científico como discurso único ${ }^{10}$. Esa denuncia deviene resistencia y deviene rechazo de la objetivación, de la abstracción y del subsumir toda otra forma de saber al de la ciencia galileana. La barbarie lleva en germen la destrucción de la cultura, su sometimiento y ulterior destrucción, que es la destrucción del hombre, la negación de la vida. El sujeto es, así, hecho objeto. Se trata del sometimiento de la naturaleza, de la vida. Es el modo de "provocación" de la técnica moderna del que hablaba Heidegger en La pregunta por la técnica. Contra esas fuerzas se lucha.

8 Domingo Moratalla, T. Op. cit., p. 9.

$9 \mathrm{lb} .$, p. 10.

10 Hart define la barbarie de Henry de la siguiente manera: "[L]a barbarie es la negación sistemática de esta vida y el esfuerzo sistemático por extinguir la conexión normativa entre la vida humana y la vida trascendental" (HART, J. "A phenomenological theory and critique of culture: A Reading of Michel Henry's La Barbarie", en Continental Phil. Rev. 32, 1999, p. 255), y sostiene que "[I]a teoría de la barbarie de Henry es verla como una profundización de los temas de Husserl en la crisis. Aquí, la cosmovisión científica post-galileana es vista como una forma de abstracción, supresión y negación dañinas del todo fenomenológico que es la vida de constituir una subjetividad trascendental" (Ib.). 
Suele indicarse, por otro lado, que La barbarie sigue un camino desmalezado por Husserl en La crisis de las ciencias europeas y la fenomenología trascendental, y viene a retomar la crítica de la reducción del mundo de la vida al mundo de la ciencia pero con un objetivo distinto al de Husserl en tanto que "no se trata de volver a una subjetividad olvidada, [sino que su] crítica a la reducción galileana es más amplia, más radical, más esencial"11. De la afirmación de que la vida es la fuente de todas las formas de cultura se sigue que distanciarse de la vida significa eliminar la cultura, y si la ciencia es extraña al arte, a la cultura y a la vida, pues ésta supone "la eliminación de la sensibilidad" y, dejada a sí misma, la ciencia "se convierte en técnica, la cual excluye toda acción subjetiva". La pretensión de Henry es, digámoslo, la de una fenomenología material que sea capaz de aprehender la vida, que sea capaz de pensar la subjetividad en sí misma. Desde estas coordenadas, ¿cómo no ver, pues, en la ideología de la barbarie un foco de amenaza sobre el cual centrar el ataque?

Aun así, y para que la tarea de reconocer el valor de la enjundia filosófica de la obra de Michel Henry no vaya en desmedro de una exposición honesta del texto, debemos insistir con una aclaración que hicimos al comenzar: Ante un texto como La barbarie, surge inmediatamente la pregunta de si uno no está frente a una muestra de irracionalismo teñido de ese tinte nostálgico que uno ya puede reconocer en la poética de textos como Die Frage nach der Technik. Cabe preguntarse, pues, si uno no está frente a una actitud anti-científica "de a ratos" como la que a menudo encuentra, por ejemplo, en la crítica que filósofos de comienzos del siglo XX como Husserl y Bergson hicieron de la imagen de la naturaleza propuesta por la física moderna. Después de todo, cabe recordar que Jean Lacroix pretendió elogiar a Michel Henry al llamarlo "el nuevo Bergson". De todos modos, sin que lleguemos a negar la ingenuidad y el exceso de reducción de muchos pasajes de $L a$ barbarie, que los hay y en abundancia, encontramos muchísimos elementos interesantes en esta exposición de la filosofía de Henry, a la vez de que encontramos interesante la conversación que ésta establece con el trabajo de otros filósofos que trataron el tema: Nuevamente, la referencia a Heidegger es ineludible.

\subsection{Resumen}

Organizaremos el resto de este trabajo de la siguiente manera: En la sección segunda expondremos los elementos del libro La barbarie que encontramos principales para nuestra discusión. En la sección tercera ahondaremos en el análisis comparativo con los textos de Heidegger, y en especial con Die Frage nach der Technik (1954). Haremos

11 Domingo Moratalla, T. Op. cit., p. 11. 
esto con la intención de responder a la pregunta acerca de cuáles de los elementos que vertebran la discusión principal en La barbarie trazan líneas directas con los argumentos de la exposición célebre que Heidegger hiciera del problema de la técnica en sus escritos de los años 1940 y 1950; escritos tales como Grundbegriffe (1941), Die Frage nach der Technik (1954) y Was heisst denken? (circa 1954). Se ha señalado ya que la crítica henryana a la fenomenología retoma y continúa una crítica que ya había hecho Heidegger al rol desempeñado por la intencionalidad en Husser ${ }^{12}$. Aquí nosotros nos centraremos en otro aspecto de la relación entre Henry y Heidegger, no tan centrado en la crítica a la intencionalidad en Husserl, sino estrictamente relacionado con el tema central de La barbarie: el riesgo del saber científico como saber único, y lo que Heidegger llamaría un único modo de des-ocultamiento.

\section{Entrar en La barbarie}

\subsection{Lo nunca visto}

Así se refiere Henry a la situación a la que nos enfrenta la ideología de la barbarie: "Lo nunca visto". Se trata de una situación abismal que ya no es una de aquellas en las que

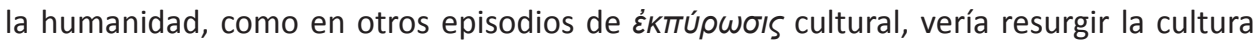
luego de una conflagración. El riesgo al que nos enfrentamos ahora, presagia Henry, es mucho peor: Ya no cabrá esperar el resurgimiento de nuevas expresiones de la cultura a partir del aquietamiento del fuego de templos pretéritos derribados, ya no se dará la situación de que verdes renuevos nazcan en las grietas pacientes de las ruinas de saberes anteriores. Por el contrario, la barbarie nos ofrece un futuro yermo, nos prepara un terreno devastado que, a diferencia de los que la humanidad enfrentó varias veces en el pasado, anticipa la muerte definitiva de la cultura, lo nunca visto. La barbarie no es el comienzo, sostiene Henry, sino que sucede ya a un estado de cultura: "Es ya la ruina". Tal es el tono apocalíptico del comienzo del libro de Henry.

"Entramos en la barbarie", nos dice. En sus palabras, "no es la primera vez que la humanidad cae en la noche"13. De hecho, muchas veces una civilización desapareció, pero "siempre hubo otra que le sucedía. Sobre las ruinas de antiguos santuarios se levantan

12 Por ejemplo: "Henry traza su mirada retrospectiva notando que Heidegger critica a Husserl porque la intencionalidad silencia el ser de la intencionalidad y la sitúa en la conciencia como en una caja" (Cfr. INVERSo, H. Fenomenología de lo inaparente, Prometeo, Buenos Aires, 2018, p. 260). 13 HeNRY M. La barbarie. Op. cit., p. 15. 
nuevos templos, más poderosos, más refinados"14. Lo que ahora ocurre es diferente, inusitado, es lo "nunca visto". Escribe:

Lo que sucede bajo nuestros ojos es bien distinto. Asistimos desde el comienzo de la era moderna a un desarrollo sin precedente de los saberes que constituyen "la ciencia" y reivindican arrogantemente este título. Por ciencia se entiende un conocimiento riguroso, objetivo, indiscutible, verdadero $^{15}$.

De las formas aproximativas de conocimiento que la habían sucedido, dice Henry, la ciencia "se distingue (...) por el poder de sus evidencias y de sus demostraciones, de sus 'pruebas', al mismo tiempo que por sus resultados extraordinarios que ha obtenido y que trastornan la faz de la tierra"16. Y en este acto de reconocimiento de los resultados de la ciencia encuentra la desgracia: "Tal trastorno (...) es también el del hombre mismo"17. Por supuesto, podría uno reaccionar aquí señalando un prejuicio infundado, el de que el trastorno de la faz de la tierra y la alteración de una naturalidad primigenia preexistente no son necesarios para la vida, para potenciarla, e incluso para expresarla en su más alto grado. Incluso, podría argüirse que el mantenimiento de la vida y su super-vivencia dependen de ese trastorno de lo natural. Pero también es claro que siempre nos podría objetar Henry que no es esa la vida de la que habla aquí, y sobre este punto ahondaremos más abajo.

\subsection{La fragmentación del saber y el riesgo de un saber único}

Nos dice Henry que el saber "ha debido fragmentarse en una proliferación de investigaciones cada una con sus propias metodologías, sus aparatos conceptuales y sus objetos"18, lo que nos invita a una primera comparación con Heidegger, quien había ya advertido no sólo de la fragmentación de las disciplinas y saberes, sino también del riesgo que tal fragmentación engendra. Para Heidegger, la técnica amenaza con la posibilidad de que todo desocultar se represente todo únicamente, de un modo único posible ${ }^{19}$.

\section{$14 \mathrm{lb}$.}

$15 \mathrm{lb}$.

$16 \mathrm{lb}$.

17 Ib., p. 16.

$18 \mathrm{lb}$.

19 Cfr., HeIDEgGer, M. "La pregunta por la técnica", en Filosofía, Ciencia y Técnica. Universitaria, Santiago de Chile, 2017. 
De manera similar, nos dice Henry, en adelante "[I]o que se cuestiona es la unidad del saber"20. Dice:

Con la interpretación de la crisis de la cultura como resultado de la multiplicación indispensable de saberes que obedecen a la voluntad de rigor y objetividad de la ciencia, se mantiene una presuposición que pasa por obvia: estos saberes, por diversos que sean, constituyen el saber único posible. (p. 16).

Y la cuestión de la singularidad de algunos saberes particulares es esencial en el análisis de La barbarie. Henry se pregunta cómo es posible que un cierto tipo de saber, aparecido a mediados del siglo XVII, en la época de Descartes y Galileo, y considerado desde entonces como el único saber ${ }^{21}$, produzca "la subversión de todos los demás valores y así de la cultura y, de esta forma, de la humanidad del hombre"22. La ciencia moderna "precipita nuestro mundo hacia el abismo", se denuncia. Pero para entender esto, Henry encuentra preciso preguntarse primero qué es la cultura. Toda cultura, nos dice, es una cultura de la vida "en el doble sentido" en que la vida constituye a la vez el sujeto de esta cultura y su objeto. Para Henry la cultura es una acción que la vida ejerce sobre sí misma y por la que se transforma a sí misma en cuanto es ella misma la que transforma y lo que es trasformado. La cultura, así, "designa la autotransformación de la vida".

Pero si la vida es este movimiento incesante de autotransformación, entonces la vida de la que se habla en La barbarie no puede tratarse de "la vida estudiada por la biología, el objeto de una ciencia: las moléculas y partículas que el científico espera encontrar a través de sus microscopios"23. Nuevamente resuena aquí Heidegger ${ }^{24}$ :

20 HenRY M. La barbarie. Op. cit., p. 16.

21 En relación a la unicidad de saberes y el surgir de una ciencia de saberes universales, hay una inversión que resulta interesante. A propósito de la cuestión de la técnica hoy, Gianni Vattimo escribe: "Solamente si todos los elementos del mundo material están sujetos a una única autoridad y no a diversas deidades, como en el politeísmo, deviene posible construir una ciencia general de la naturaleza, con leyes universales, válidas en todas artes (...). Es como decir que Newton y Galileo nunca podrían haber hecho sus descubrimientos fuera de una visión monoteísta de la naturaleza" (VATtiMO, G. Alrededores del ser. Galaxia Gutenberg, Barcelona, 2020, p. 156). La inversión se da aquí en tanto la unicidad de saberes aparece como condición de la aparición de la ciencia galileana, y no en la relación causal inversa según la cual la ciencia galileana demanda la instauración de su saber como el único.

22 HenRY M. La barbarie. Op. cit., p. 17.

23 lb., p. 18.

24 Esto se relaciona también con lo que para Heidegger es el problema de la imposibilidad de un metalenguaje en la ciencia; por ejemplo: "El que toda ciencia, cuanto tal, es decir en cuanto la ciencia que es, le sean inaccesibles sus conceptos fundamentales está en conexión con el hecho de que ninguna ciencia puede decir algo sobre ella misma con sus propios medios científicos. (...) [L]o 
Nosotros estamos fuera de la ciencia. Y en lugar de estar en ella nos encontramos, por ejemplo, ante un árbol en flor (...). [E]chamos todo a perder tan pronto como, al unísono con la ciencia física, la fisiología y la psicología, junto con la filosofía científica, con todo despliegue de sus materiales y pruebas, afirmamos que propiamente no percibimos ningún árbol, sino que en realidad percibimos un vacío donde se hallan dispersas cargas eléctricas, que circulan de aquí para allá a gran velocidad ${ }^{25}$.

En su Carta sobre el humanismo Heidegger se expresaba en el mismo sentido: "[H]oy tendemos a renunciar al árbol floreciente en favor de conocimientos físicos y fisiológicos supuestamente superiores"26. También:

Que la fisiología y la química fisiológica puedan investigar al ser humano en su calidad de organismo, desde la perspectiva de las ciencias naturales, no prueba en modo alguno que en eso "orgánico", es decir, en el cuerpo científicamente explicado, resida la esencia del hombre ${ }^{27}$.

De igual manera, la vida de la que habla Henry en La barbarie no debe confundirse "con el objeto del saber científico" ${ }^{28}$. La "vida verdadera", para Henry, es "la vida fenomenológica absoluta, cuya esencia consiste en el hecho mismo de sentirse o de experimentarse a sí misma y no es nada más"29. Para Henry es precisamente eso que llamamos la subjetividad. Es a partir de esta distinción del término vida, de carácter definitorio, que Henry afirma que la cultura no tiene originariamente y en sí misma nada que ver con la ciencia y no proviene en modo alguno de ella ${ }^{30}$.

Advierte que si "decimos que [la] propiedad extraordinaria de experimentarse a sí mismo es un saber (...) importa precisar de qué clase de saber se trata" ${ }^{31}$. No es el saber científico, insiste, dado que ése "es objetivo por principio". Al respecto, escribe:

que sea la biología no puede decirse nunca biológicamente" (Cfr. HEIDEGGER, M. On time and being. University of Chicago Press, Chicago, 2002, p. 302-La traducción es del autor). De hecho, podemos decir que para Heidegger la cuestión de la ciencia es un problema de metalenguaje.

25 HeIDEgGER, M. ¿Qué significa pensar?. Trotta, Madrid, 2005, pp. 34-35.

26 HeIDEgGer, M. Carta sobre el humanismo. Alianza, Madrid, 2013.

27 HeIDEgGeR, M. ¿Qué significa pensar?. Op. cit., pp. 32-33.

28 HenRY M. La barbarie. Op. cit., p. 20.

$29 \mathrm{lb} .$, p. 21.

30 Cfr. Ib., p. 20.

31 lb., p. 21. 
[La] pretensión de superar la particularidad y la relatividad de lo "subjetivo" (...) ha jugado un papel decisivo en la época de Galileo, en el nacimiento de la ciencia moderna, es decir, de la ciencia matemática de la naturaleza ${ }^{32}$.

Nos dice que esta ciencia galileana de la naturaleza, en el conocimiento de este mundo, se hace precisamente abstracción de las cualidades sensibles y, de una manera general, de todo lo que es tributario de la subjetividad para no retener, como existiendo realmente, sino las formas abstractas del universo espaciotemporal. Estas formas [abstractas] se prestan entonces a una determinación geométrica que es la misma para cada espíritu ${ }^{33}$.

El saber que consiste en esa propiedad de experimentarse a sí mismo no tiene nada que ver, pues, con esa abstracción.

Sin detenerse en la pregunta por el origen de esa necesidad de abstracción, Henry sostiene que el destierro de la subjetividad nace de esa pretensión de universalidad de la ciencia, pretensión que lleva a la abstracción, i.e. a la reducción de la vida a formas abstractas que es necesaria para lograr ese extracto mínimo, desprovisto, pálido que es lo universalizable. La esfera de la subjetividad es la "de las sensaciones, de las opiniones, de los pensamientos personales, [y] descansa sobre la naturaleza del espíritu" ${ }^{14}$. En este sentido, Henry reivindica la crítica de Husserl a la ideología cientificista y positivista de nuestra época; escribe: "Las determinaciones geométricas, a las que la ciencia galileana intenta reducir el ser de las cosas, son idealidades"35, y sostiene que son incapaces de dar cuenta del mundo sensible, subjetivo y relativo en el cual se desarrolla nuestra actividad cotidiana. El siguiente párrafo describe su tesis con claridad:

[E]n cuanto idealidades, las determinaciones geométricas y matemáticas de las que hacen uso las ciencias de la naturaleza suponen la operación subjetiva que las produce y sin la cual no existirían: no hay en la naturaleza ni número ni cálculo, ni suma ni resta, ni recta ni curva: son significaciones ideales que encuentran su origen absoluto en la conciencia que las crea, en el sentido estricto de la palabra, y a la que se puede llamar, respecto de ellas, conciencia trascendental. $\mathrm{Si}$, pues, las idealizaciones geométricas y matemáticas provienen de la subjetividad es que, lejos de reducir ésta a no ser más que una apariencia, el mundo de la ciencia encuentra en ella,

$32 \mathrm{lb}$.

33 lb., p. 22.

$34 \mathrm{lb}$.

$35 \mathrm{lb}$. 
al contrario, el principio que lo engendra continuamente como la condición permanente de su propia posibilidad ${ }^{36}$.

Puede decirse que Henry parece caer aquí en un dualismo irreconciliable con su propia crítica inicial. La ausencia de la pregunta acerca del origen de la necesidad de la universalidad y la abstracción como su condición de posibilidad es el punto débil de su análisis crítico. Lejos de identificar y analizar el recorrido dialéctico que desemboca en la aparición de la barbarie y la promulgación de la ideología que denuncia, Henry reduce el aparecer de tal ideología en nuestro mundo a la categoría de acontecimiento externo, de irrupción. La cultura -y eso se ve con más fuerza al final de su libro- se yergue, según su visión, como una suerte de résistance, un reservorio moral clandestino y último.

\subsection{Una defensa desde la angustia}

Insistir con el carácter siempre foráneo de la barbarie, de esa ideología tecnocrática que subsume todo otro saber hasta erigirse como el único, de la necesidad de universalidad que lleva a la abstracción y que pertenece, aunque no exclusivamente, al cientificismo deshumanizante, no le permite a Henry identificar con lucidez y distancia suficiente aquellos rasgos de la barbarie que son nuestros. Y uno podría aquí ensayar una defensa desde la angustia: Ante la pregunta acerca del origen de la necesidad de esa universalidad, de ese imperativo unificador y generalizador de las verdades, de ese deseo de reduccionismo, de esa insistencia con la abstracción, la respuesta común sería que ese origen se encuentra en el deseo de dominación, o incluso en la mera disposición, en la curiosidad. Pero la empresa científica no nace de ello. Por el contrario, su origen no es nada feliz; la ciencia nace de la angustia, una angustia que es tan nuestra, subjetiva y primera como todo otro sentir de los que nos dirigen hacia el acto creativo. Negar la angustia como aquello que engendra la empresa científica y reducir esta última a una sucesión de actos volitivos de dominación -o a satisfacciones de la curiosidad- es desconocer lo que de creativo hay en la ciencia ${ }^{37}$. Sin desdeñar lo que de lúdico puede haber en la búsqueda ni dejar de reconocer el carácter fruitivo de la obtención de una respuesta, si se quiere investigar el verdadero origen de la ciencia no habrá que buscarlo en ese deseo de dominación, que no es sino una imagen feble del hacer científico, sino que habrá que buscarlo en la angustia. Ahora bien, Henry podría replicar aquí lo siguiente: Esa angustia nace, de hecho, de la sensación de foraneidad que experimentamos ante el mundo, de ese no ser parte

36 lb., p. 23.

37 Más adelante, sobre el final de esta sección, volveremos sobre la relación entre la ciencia y otros actos creativos, como los artísticos. 
del escenario que se monta a nuestro alrededor y, por tanto, nace del reconocimiento del mundo, de la exterioridad, y entonces no es primero. Para Henry lo primero es la vida, esa vida subjetiva de la que hablábamos arriba: "[E]sta vida subjetiva no crea solamente las idealidades y las abstracciones de la ciencia" sino que "da primero forma al mundo de la vida en medio del cual se desarrolla nuestra existencia concreta"38. La palabra "primero" aquí cabe ser resaltada; es importante para él en tanto investigador del fundamento último del aparecer.

\subsection{La fenomenología como método y tópico}

Volviendo a Husserl, y sin ocultarlo, Henry escribe: "Cada percepción de una cara de un cubo (...) remite a las percepciones potenciales de las otras caras todavía no percibidas, según un juego de relaciones indefinidas"39, y agrega que lo mismo ocurre con "todo objeto general, toda formación trascendente, que siempre implica una operación sintética específica de la subjetividad trascendental, sin la cual no existiría"40. Es tarea de la filosofía, dice Henry, evidenciar la actividad incansable de la conciencia que percibe el mundo, que concibe "las idealidades y las abstracciones de la ciencia", que imagina, que recuerda, produciendo así todas las representaciones irreales que no cesan de acompañar el curso de nuestra vida real ${ }^{41}$. La concepción implícita de la conciencia, de la "phénoménalité de los fenómenos", se encuentran, dice Henry, en el trasfondo de la mayor parte de las filosofías y en la ciencia misma. En Kant, por ejemplo, la posibilidad de la experiencia es el conjunto de las condiciones gracias a las cuales pueden dársenos los objetos. Luego,

[e]sta posibilidad de relacionarse con los objetos, de trascenderse hacia ellos con el fin del alcanzarlos, es, en la fenomenología husserliana, la intencionalidad, que define el fondo de la conciencia misma, su poder de mostración y de exhibición, es decir, la phénoménalité misma ${ }^{42}$.

Sostiene que el rechazo de la fenomenología post-husserliana de los conceptos de subjetividad y de conciencia es justamente el rechazo de todo lo que no se reduciría a esta "eclosión primitiva del Afuera donde se sitúa el Objeto"43. Insistiendo con esto, dice que el rasgo distintivo del saber científico es su objetivación. Lo que es verdaderamente

38 HeNRY M. La barbarie. Op. cit., pp. 22-23.

39 lb., p. 24.

$40 \mathrm{lb}$.

$41 \mathrm{Cfr}$. Ib.

42 lb., p. 25.

$43 \mathrm{lb}$. 
científico, apunta Henry, es tal que es conocido por todo espíritu; pero "la objetividad del saber científico en el sentido de su universalidad, descansa sobre la objetividad ontológica de la que acabamos de hablar: sobre el hecho de que lo que es verdadero debe ser demostrado"44. Esta observación es fundamental y sobre ella cabe detenerse, en tanto deja en claro los dos sentidos en los que el de Henry es un análisis fenomenológico. Por un lado, su análisis cumple con poner entre paréntesis al acervo de prejuicios con el que uno se enfrenta a la cuestión de la ciencia moderna en nuestros tiempos; dirá que

es forzoso rechazar, de entrada, el conjunto de los prejuicios de nuestro tiempo, a saber, la creencia de que no solamente el saber científico es el más importante sino que en realidad es el único saber verdadero; que saber quiere decir ciencia, es decir, este tipo de saber matemático introducido en la época de Galileo ${ }^{45}$.

Por otro lado, la afirmación de arriba: "lo que es verdadero debe ser demostrado", lleva a Henry a argumentar que el científico está, antes que nada, en contacto con el aparecer. Así, el rótulo "fenomenológico" en referencia al análisis efectuado en La barbarie es bisémico. La fenomenología entra de dos maneras aquí: de manera metodológica y de manera tópica, entendiendo por esta última la manera en la que pone de relieve el aparecer como tal en la vida cotidiana del científico en su laboratorio. El científico debe convencerse y, en tanto portador de un saber universal, debe convencer, debe demostrar, debe dar evidencias, y esas evidencias "aparecen". El aparecer es, entonces, ineludible y primero, incluso en la vida del científico. Para aclarar este punto, nos propone observar a un estudiante de biología: "[N]o es el saber científico el que le permite [al estudiante] adquirir el saber científico contenido en el libro; no es gracias a él por lo que mueve sus manos o sus ojos, o concentra su espíritu"46. Esto lo lleva a un terreno que Henry conoce bien: el de la corporalidad, el cuerpo como acción pero también como pathos; el estudiante, el científico, ante todo, mueve sus manos para voltear las hojas, mueve los ojos al leer. Hay algo "primero", algo "antes" del saber científico, incluso en ese alcanzarlo ese "hacerse" de él. Dice: "El saber científico es abstracto: es la intuición intelectual de cierto número de significaciones ideales. Pero el acto de mover las manos no es nada abstracto"47. Así, el saber científico es objetivo, ante todo en el sentido de que es conocimiento de una objetividad, pero "la capacidad de unirse al poder de las manos [al

44 lb., p. 26.

45 Ib., p. 27.

46 Ib., p. 28.

$47 \mathrm{lb}$. 
leer] no es sino su subjetividad radical"; agrega: "[E]n la inmanencia de su subjetividad radical (...) es posible el poder de las manos"48.

\subsection{Con-ciencia}

La de traer el problema del cuerpo a la discusión no es la única operación de este tipo que Henry realiza en La barbarie. Hace algo similar al traer a la discusión el problema de la crítica a la conciencia, otro territorio que le es familiar. Henry elige armas en este duelo con la barbarie. Lo hace al afirmar que "el saber científico es homogéneo con el saber de la conciencia en general"; más aún: "simplemente lo prolonga"49. Así, la crítica a la conciencia como fundamento se transfiere a la del saber científico como saber fundamental. Esto lo habilita, pues, a hacer una crítica a la forma del saber de la ciencia. Dirá: "[e]l saber científico (...) no es más que una modalidad del saber de la conciencia"50. El saber científico, sostiene Henry, no es más que una modalidad del saber de la conciencia, es decir, de la relación con el objeto ${ }^{51}$. Ese saber no es el saber de la vida, en tanto la vida sólo la vida se relaciona consigo misma. Insiste: "De lo que es la vida (...) la ciencia no tiene ni idea, en absoluto se preocupa de ella, no tiene relación alguna con ella y nunca la tendrá"52. Una vez más, resuena aquí Heidegger: "Que la fisiología y la química fisiológica puedan investigar al ser humano en su calidad de organismo (...) no prueba en modo alguno que en eso (...) resida la esencia del hombre"53.

\subsection{La ciencia y el juicio}

Henry dedica uno de los capítulos de La barbarie enteramente al estudio de la ciencia juzgada según el criterio del arte. Henry se propone allí a discutir su tesis: La ciencia excluye la sensibilidad porque de eso depende la demarcación de su campo de dominio. En sus propias palabras,

[e]l arte, en efecto, es una actividad de la sensibilidad, la realización de sus poderes, mientras que, con la eliminación de las cualidades sensibles de

48 Ib., p. 29.

$49 \mathrm{lb} .$, p. 26.

50 lb., p. 29.

$51 \mathrm{Cfr} . \mathrm{lb}$.

52 lb., pp. 35-36.

53 HeIDEgGer, M. Carta sobre el humanismo. Op. cit., p. 33. 
la naturaleza, la ciencia moderna define su campo propio y se define a sí misma por la exclusión de esta misma sensibilidad ${ }^{54}$.

Comienza su análisis de la ciencia en relación al criterio del arte con la impronta provocadora que caracteriza al resto de su libro. Declara: "La ciencia, en cuanto tal, no guarda relación alguna con la cultura" ${ }^{15}$. No obstante, inmediatamente después de lanzar su frase controversial ${ }^{56}$ aclara que esto "no legitima en sí misma alguna apreciación peyorativa que descalifique a la ciencia, ninguna condena"57. Esto recuerda al movimiento que Heidegger realiza en Was heisst denken?, donde, al instante de espetar su famosa aserción: "Die Wissenchaft denkt nicht" (La ciencia no piensa), aclara que "[e]l hecho de que la ciencia no pueda pensar no es una carencia sino una ventaja"58. Al igual que Heidegger, Henry esconde la mano luego de lanzar la piedra. Y al igual que Heidegger, sostiene que "[e]l filósofo tiene el deber de intervenir sólo cuando el dominio de la ciencia es comprendido como el único dominio de ser verdaderamente existente"59. Lo que Henry critica no es el saber científico per se, sino "la ideología que hoy en día se le une y según la cual él es el único saber posible (...), según la cual saber quiere decir ciencia"60. No obstante, dado que al mismo tiempo reconoce que esa unicidad del saber científico está grabada a fuego en la ciencia misma, en su pretensión de universalidad, su crítica es una crítica a la ciencia, sin más. Para él, la ciencia y arte, que es decir la ciencia y la sensibilidad, "caen la una fuera de la otra por efecto de una heterogeneidad tan radical de sus dominios respectivos"61. Y para que no se juzgue esta distinción que él establece como si se tratase de un antagonismo ingenuo que puede ser resuelto como cualquier falsa antinomia, se adelanta a replicar: "No nos dejaremos perturbar por la objeción superficial según la cual sería posible encontrar, en el interior mismo del trabajo científico y de sus producciones, muchas 'bellezas'"62. Digamos aquí que Henry parece manejar aquí una idea rudimentaria de estética en el acto científico. Mientras habla de "la armonía de las representaciones" que nos procuran las producciones de la ciencia y piensa en composiciones regulares de la naturaleza, deja afuera otras nociones de estética, acaso más elaboradas y ajustadas

54 HeNRY M. La barbarie. Op. cit., p. 41.

$55 \mathrm{lb}$.

56 Luego diría también que "la ciencia elimina la vida", todo lo que de ella proviene y todo lo que a ella remite.

$57 \mathrm{lb}$.

58 HeIDEgGER, M. ¿Qué significa pensar?. Op. cit., pp. 19-20.

59 HenRy M. La barbarie. Op. cit., p. 41. Cfr. Heidegger, M. "La pregunta por la técnica". Op. cit. 60 HenRY M. La barbarie. Op. cit., p. 41.

$61 \mathrm{lb}$.

$62 \mathrm{lb}$. 
a la creación científica. En términos hegelianos, podemos decir que Henry restringe su análisis de los modos en los que se expresa la forma de la naturaleza a la regularidad y a la armonía, dejando de lado formas más elevadas de belleza tales como lo que Hegel llama Gesetzmässigkeit, i.e. el modo en el que se presenta en la naturaleza la belleza exterior de la forma abstracta mediante la conformidad a una ley ${ }^{63}$. El análisis estético que Henry hace de la ciencia es, usando sus propios calificativos, superficial; tan superficial como lo es la objeción a la que se adelanta y a la que correctamente replica ${ }^{64}$. La belleza de las creaciones científicas no se agota en la regularidad sutil que exhibe la estructura microscópica de los cristales cuasiperiódicos o ejemplos de este estilo, sino que se eleva por sobre todo ello y encarna en la arquitectura misma del edificio conceptual de las teorías generales, universales y abstractas que Henry acusa de opresoras del arte. La dialéctica de la estética de la ciencia es más abstrusa, y es tal que son precisamente esos atributos de generalidad, concisión y universalidad -antitéticos de lo que Henry llama vida- los que, en síntesis, dotan a las construcciones teóricas de la ciencia moderna de su contenido de belleza. Ante la arquitectura teórica de la teoría general de la relatividad, i.e. la teoría universal de la gravitación de Einstein, el sentimiento de belleza que se experimenta es, sobre todo, irreducible al análisis de otras formas de lo bello.

Más interesante que el análisis estético de Henry es su análisis genealógico, i.e. su pregunta por el surgimiento del arte: "¿[P]or qué el arte surge necesariamente en el interior de la experiencia humana como una de las formas fundamentales de toda cultura?"65. Sin evitar el circunloquio, se responde: "[T]oda cultura incluye en ella al arte como una de sus dimensiones esenciales [porque] pertenece por su naturaleza al mundo de la vida donde viven los hombres, porque todo hombre en cuanto habitante de este mundo es potencialmente un artista"66. Henry encuentra en esto el significado de que "la ciencia ponga fuera de juego a la sensibilidad". Pero tal exclusión es imposible, nos dice; "es imposible, puesto que (...) la sensibilidad es la condición trascendental de todo lo que es susceptible de recibir la forma de un 'mundo'. La sensibilidad sólo es eliminada del mundo científico. Y ésta es la razón de que este mundo sea abstracto"67.

63 V. Hegel G. W. F. Estética I, Losada, Buenos Aires, 2008, pp. 115-136.

64 Sobre estas composiciones armónicas y regulares de naturaleza Henry nos dice, con acierto, que las mismas son, aun así, siempre juzgadas con las leyes de la estética y no con las de la ciencia. 65 Henry M. La barbarie. Op. cit., p. 43.

66 Ib., p. 45.

$67 \mathrm{lb}$. 


\section{La cuestión de la técnica}

\subsection{La reducción al mundo-de-la-ciencia}

Así entiende Henry la barbarie: La ideología que, desde el surgimiento de la ciencia galileana, promulga al saber de esa ciencia como saber único, como la verdad, como lo que Heidegger denominaría único modo de desocultar; subordinando así a todos los otros saberes, los que forma la cultura: la ética, la religión, el arte; llevándolos al riesgo de su extinción definitiva. Esta eliminación de la cultura es, para Henry, la eliminación de la vida.

La ciencia a la que se refiere Henry es la ciencia matemática de la naturaleza, que hace abstracción de la sensibilidad. Y él advierte que "la ciencia sólo puede hacer abstracción de la sensibilidad haciendo primero abstracción de la vida"68; esto es decir que la vida que "es rechazada" de la temática de la ciencia. La descripción científica de los colores en términos de longitudes de onda de la porción visible del espectro electromagnético nada tiene que ver con la sensación de color ${ }^{69}$. La descripción científica del color y la sensación de color están separadas por un "abismo ontológico". Dirá Henry como corolario: "La sensación se siente a ella misma"70. Estas digresiones le llevan a concluir que "la ciencia elimina la vida y, con ella, todo lo que de alguna manera proviene de ella y a ella remite"71. La pretensión de la ciencia de reducir el mundo de la vida a un mundo de idealidades y de abstracciones físico-matemáticas, nos dice Henry, descansa en la ilusión previa de que las propiedades sensibles de este mundo son las [las de la ciencia] y le pertenecen propiamente y que, puesto que el color está en la naturaleza y no en el alma, se puede apresar su ser natural ${ }^{72}$. Y exhorta:

Prohibir (...) la reducción del mundo-de-la-vida al mundo-de-la-ciencia sólo puede ser hecho por un pensamiento capaz de captar el mundo-de-la-vida en su especificidad, (...) en su irreductibilidad al mundo y a todo mundo posible $^{73}$.

68 lb., p. 63.

69 Nuevamente podemos encontrar aquí reminiscencias de las críticas que Bergson, Husserl y otros hicieron a la descripción del tiempo propuesta, por ejemplo, por la teoría de la relatividad especial de Einstein, al denunciar que tal descripción de un tiempo abstracto nada tenía que ver con el tiempo que es dado a nuestras sensaciones y que experimentamos en nuestra vida.

70 HENRY M. La barbarie. Op. cit., p. 63.

71 lb., p. 64.

72 Cfr. Ib., p. 65.

$73 \mathrm{lb}$. 
En este punto Henry aprovecha para entrelazar su discusión de la reducción al mundode-la-ciencia con su crítica al papel de la "conciencia del mundo" en la fenomenología de Husserl. Dice:

Aquí se nos hace patente la insuficiencia del proceder ya clásico de la fenomenología que, tomando a contrapié a la ciencia galileana, ejecuta el cuestionamiento regresivo del mundo-de-la-ciencia al mundo-de-la-vida, y de éste a la conciencia de este mundo. Pues la conciencia del mundo en cuanto "conciencia de", en cuanto intencionalidad, (...) no permite todavía el ensimismamiento de la sensación y que de esta manera ésta llegue a $\operatorname{ser}^{74}$.

Henry sostiene que "[l]a ciencia que se cree sola en el mundo y que se comporta como tal se convierte en la técnica"75. Esto permite ver que Henry coincide con Heidegger en dos puntos al respecto de la relación entre ciencia y técnica; por un lado, en que la ciencia y la técnica son dos cosas distintas; por el otro, en que, aunque distintas, la primera lleva en germen a la segunda, anticipándola. La técnica es, para Henry, "el conjunto de operaciones y de transformaciones que extraen su posibilidad de cualquier otra forma de saber"76, consideración que lo hace caer nuevamente en el riesgo de la circularidad. También al igual que Heidegger, Henry encuentra imperioso hacerse la pregunta sobre la "esencia de la técnica", que, como Heidegger aclaraba77, "no es una cuestión de la técnica"78. Henry nos dice: "La esencia de la técnica, en su doble relación, positiva para con la ciencia, negativa para con la vida, es difícil de captar. Hay que proponer una elucidación sistemática de la misma"79.

\section{$74 \mathrm{lb}$.}

75 lb., p. 67.

$76 \mathrm{lb}$.

77 Por ejemplo: "Preguntamos por la técnica y quisiéramos, así, preparar una relación libre con ella. Libre es la relación cuando abre nuestro ser-ahí (Dasein) a la esencia de la técnica. Si nosotros correspondemos a eso, entonces podremos experimentar la técnica en su delimitación. La técnica no es igual que la esencia de la técnica" (HEIDEGGER, M. "La pregunta por la técnica". Op. cit., p. 55). $78 \mathrm{Cfr}$. Ib.

79 HenRY M. La barbarie. Op. cit., p. 68. 


\subsection{La téchne}

El paralelismo con El problema de la técnica de Heidegger se acentúa cuando habla Henry de las causas y los medios en función de dar una definición de la técnica. Escribe: "Y quien dice medios dice fines" 80 . Continúa:

Desgraciadamente, de estos "intereses superiores" de la "humanidad" misma, es decir, de la esencia de la vida, tanto la ciencia como la técnica que de ella resulta no saben estrictamente nada y no los tienen nada en cuenta (...) Por ello, si a propósito de la técnica, se habla de "medios", hay que reconocer que se trata de medios muy particulares, los cuales ya no están al servicio de ningún fin diferente de sí mismos, sino que son ellos los que constituyen el "fin"81.

Es imposible aquí no traer las palabras de Heidegger:

[L]a técnica es un medio para un fin (...) A lo que la técnica es pertenece el elaborar y utilizar instrumentos, aparatos y máquinas, pertenece este elaborar y utilizar mismo, pertenecen las necesidades y fines a los que sirven. El todo de estas organizaciones es la técnica. Ella misma es una organización, dicho en latín: un instrumentum. (...) [T]también la técnica moderna es un medio para un fin. Por eso, la representación instrumental de la técnica determina todos los esfuerzos para llevar al hombre a la justa relación con la técnica. Todo estriba en manejar la técnica, en cuanto medio, de la manera adecuada. Pero, suponiendo que la técnica no sea ningún simple medio, ¿qué pasa entonces con el querer dominarla? Pero, nosotros dijimos que la determinación instrumental de la técnica era correcta. (...) La técnica no es, pues, simplemente un medio. La técnica es un modo del desocultar. Si prestamos atención a eso, entonces se nos abrirá un ámbito completamente distinto de la esencia de la técnica. Es el ámbito del desocultamiento, esto es, de la verdad, del veri-ficar ${ }^{82}$.

La historia esencial de la técnica, dice Henry, "no es una historia en verdad esencial más que si se remonta al origen de la téchne, es decir a su verdadera esencia"83. Henry se

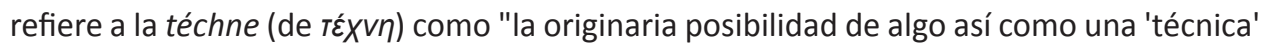

$80 \mathrm{lb}$.

$81 \mathrm{lb}$.

82 HeidegGeR, M. "La pregunta por la técnica". Op. cit., pp. 56-60.

83 HENRY M. La barbarie. Op. cit., p. 69. 
y también a las diferentes fases que ésta iba a revestir en su desarrollo"84. La palabra téchne es también cara para Heidegger en su discusión sobre la esencia de la técnica:

Con respecto a la significación de réXv $\eta$ debemos observar dos cosas. De

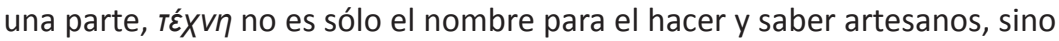
también lo es para el arte más elevado y para las bellas artes ${ }^{85}$.

En relación a esto, Henry dirá que uno se encuentra en "una situación extraordinaria", dado que la esencia original de la réxv $\eta$ que debemos tomar en consideración "para comprender las formas diversas de la técnica y fundamentalmente la esencia de la técnica moderna que hace abstracción de la vida, es la vida misma"86. Esto se debe a que "técnica" designa de una manera mucho más general el "saber-hacer". "Todo saber-hacer, sea el que sea y cualesquiera que sean sus formas, lleva implícito este saber original que encuentra su esencia en el hacer y en último extremo en la subjetividad de éste"87, dirá. "El saber-hacer original es la praxis" y es en la vida donde la praxis se conoce, es en ella "donde es el saber-hacer original constitutivo de la esencia original de la técnica"88. Henry regresa así sobre la cuestión del cuerpo: Es en el cuerpo, su movimiento, su esfuerzo, en el

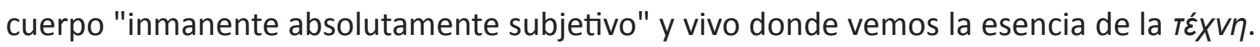
Por eso dice Henry que el mundo es siempre ante todo el mundo-de-la-vida, incluso antes de ser un mundo sensible, "ese mundo en cuanto correlato de un movimiento, en cuanto cuerpo-apropiado"89. La representación de la praxis, dice Henry, suscita la ideología que interpreta la técnica como la transformación instrumental de la naturaleza por el hombre en función de fines por él dispuestos. Concluye, pues:

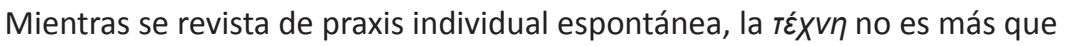
la expresión de la vida, la efectuación de los poderes del cuerpo subjetivo y por ello de las formas primeras de la cultura. (...) En las formas superiores de la cultura, como el arte, la ética o la religión, que también son modos de la

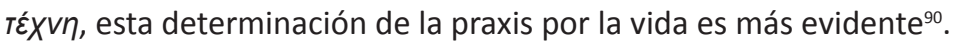

$84 \mathrm{lb} .$, p. 69. Sobre el empleo que hace Henry de la palabra téchne, dice Hart: "Henry analiza la relación ontológica original de la téchne con el compromiso de la naturaleza humana (...) Techné así es la forma en que el ser humano usa la Tierra para cambiar la Tierra de infinitas formas (HART, J. Op. cit., pp. 265-266).

85 HeIdegGer, M. "La pregunta por la técnica". Op. cit., p. 61.

86 HeNRY M. La barbarie. Op. cit., p. 69.

$87 \mathrm{lb}$.

$88 \mathrm{lb}$.

89 lb., p. 72.

90 lb., p. 73. 
Había escrito Heidegger ya al respecto:

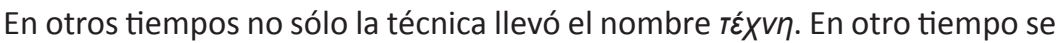

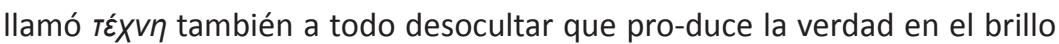

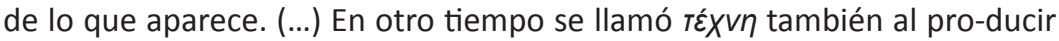

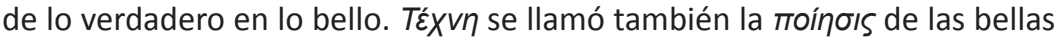
artes. (...) Al comienzo del destino occidental se alzaron las artes en Grecia a la más elevada altura del desocultar a ellas confiado ${ }^{91}$.

No nos es sencillo ni necesario ocultar la operación que intentamos aquí: la de establecer lazos directos entre la forma en la que Henry analiza en La barbarie la relación entre ciencia moderna y técnica moderna y la forma del discurso de Heidegger en Die Frage nach der Technik. La discusión de la técnica en relación a las causas, la identificación del riesgo de la instauración de un dado saber como único modo del desocultar, y la introducción de la noción de réxv comparación de estos textos. Aun así, podemos concluir de esta comparación que $L a$ barbarie no se trata meramente de una paráfrasis pesimista del ensayo heideggeriano. Por el contrario, podemos incluso advertir en La barbarie un tono esperanzador, aunque llegue éste sobre el final del libro. Como tantos otros territorios, los textos también se van fragmentando a medida que acaban, y en el caso de La barbarie esos fragmentos nos permiten reconstruir una imagen de la cultura como célula residual, aún viva, subversiva, agazapada, underground, latente. El optimismo que en Die Frage nach der Technik viene de la mano del arte como "lo salvador" en el momento que Heidegger reclama para sí las palabras de Hölderin, en La barbarie aparece como una suerte de résistance de la cultura.

\section{Referencias bibliográficas.}

BeRnet. R. "An intentionality without subject or object?". En Man and World 27, 1994. Pp. 231-255.

Domingo Moratalla T. "Introducción" en Henry, M. La barbarie. Tr. T. Domingo Moratalla. Caparrós, Madrid, 2006. Pp. 7-14.

HART, J. "A phenomenological theory and critique of culture: A Reading of Michel Henry's La Barbarie". En Continental Phil. Rev. 32, 1999. Pp. 255-270.

Hegel, G. W. F. Estética I. Tr. H. Giner de los Ríos. Losada, Buenos Aires, 2008 [Vorlesungen über die Asthetik, 1835].

91 HeIDEgGeR, M. "La pregunta por la técnica". Op. cit., p. 77. 
Heidegger, M. Conceptos fundamentales. Tr. M. E. Vásquez García. Altaya, Barcelona, 1997 [Grundbegriffe, 1941].

HeIDEgGeR, M. On time and being. Tr. J. Stambaugh. University of Chicago Press, Chicago, 2002 [Zeit und Sein, 1962].

HeIDEgGeR, M. ¿Qué significa pensar?. Tr. Raúl Gabás. Trotta, Madrid, 2005 [Was heisst denken?, 1954].

Heidegger, M. Carta sobre el humanismo. Tr. A. Leyte y H. Cortés. Alianza, Madrid, 2013. Heidegger, M. "La pregunta por la técnica". En Filosofía, Ciencia y Técnica. Ed. J. Acevedo. Tr. F. Soler Grima. Universitaria, Santiago de Chile, 2017 [Die Frage nach der Technik, 1954]. Henry, M. La barbarie. Tr. T. Domingo Moratalla. Caparrós, Madrid, 2006 [La barbarie, 1987].

Henry, M. Ver lo invisible. Tr. M. Tabuyo y A. López. Siruela, Madrid, 2008 [Voir l'invisible. Sur Kandinsky, 1988].

Henry, M. Fenomenología material. Ed. M. García-Baró. Tr. F. J. Teira Vilar. Encuentro, Madrid, 2009 [Phénoménologie matérialle, 1990].

INVERSO, H. Fenomenología de lo inaparente. Prometeo, Buenos Aires, 2018.

Vattımo, G. Alrededores del ser. Tr. M. T. Oñate y Zubia. Galaxia Gutenberg, Barcelona, 2020. 